\title{
2 UPTAKE OF SLE AUTOANTIBODIES BY PODOCYTES
}

Anja Hillmann, ${ }^{1,2}$ Hedda Wardemann, ${ }^{3}$ Thomas Pap, ${ }^{2}$ Annett Jacobi ${ }^{1}{ }^{1}$ Department of Internal Medicine D, Rheumatology, University Hospital Münster, Münster, Germany; ${ }^{2}$ Institute of Experimental Musculoskeletal Medicine, University Hospital Münster, Münster, Germany; ${ }^{3}$ Max Planck Institute for Infection Biology, Berlin, Germany

10.1136/annrheumdis-2011-201234.2

Background and objectives Systemic lupus erythematosus (SLE) is an autoimmune disease, resulting in inflammation and tissue damage in several organs. This autoimmune reaction is caused by antibodies against dsDNA (adsDNAabs). Most patients with SLE develop joint pain and about 70\% develop renal symptoms, partly with lupus nephritis leading to proteinuria. It is still unclear why the kidney and especially the podocytes (filtrating cells of the renal glomerulus) are involved in such a dramatic manner during SLE.

Methods Anti dsDNA antibodies were isolated from SLEpatients, cloned and produced recombinantly in HEK293 cells. Podocytes were treated with equal amounts of different adsDNAabs and control antibodies in time and concentration 
dependent experiments and examined by immunofluorescence. To investigate the degradation mechanism, cells were treated with Bafilomycine A or MG132 at different time points of co-cultivation with the antibodies.

Results Cultivated podocytes take up adsDNA-antibodies selectively and aggregate them in cytosolic speckles. The uptake of adsDNAabs occurs in a concentration and time dependent manner. The authors found podocytes with up to 80 aggregates per cell. The aggregates are eliminated over time in media without adsDNAabs (recovery experiment). The authors could show that the adsDNAabs are degraded by autophagy, which leads to a better survival of the cells. The number of aggregates after the inhibition of autophagy rises significantly up to twofold. The number of aggregates of podocytes treated with a control antibody is almost zero.

Conclusion Podocytes are postmitotic and highly complex cells. Loss of these cells leads to kidney injury and proteinuria. During lupus-nephritis, podocytes are destroyed. The authors could show that these cells take up SLE-antibodies selectively and are harmed by these antibody-aggregates. The authors could show that induction of autophagy can help to eliminate the aggregates. This should be considered for future therapeutic approaches in lupus nephritis. 